\title{
Global Attractivity of an Integrodifferential Model of Mutualism
}

\author{
Xiangdong Xie, ${ }^{1}$ Fengde Chen, ${ }^{1}$ Kun Yang, ${ }^{2}$ and Yalong Xue ${ }^{2}$ \\ ${ }^{1}$ Department of Mathematics, Ningde Normal University, Fujian 352100, China \\ ${ }^{2}$ College of Mathematics and Computer Science, Fuzhou University, Fuzhou, Fujian 350116, China
}

Correspondence should be addressed to Xiangdong Xie; ndsyxxd@163.com

Received 12 January 2014; Revised 8 March 2014; Accepted 9 March 2014; Published 1 April 2014

Academic Editor: Yongli Song

Copyright (C) 2014 Xiangdong Xie et al. This is an open access article distributed under the Creative Commons Attribution License, which permits unrestricted use, distribution, and reproduction in any medium, provided the original work is properly cited.

Sufficient conditions are obtained for the global attractivity of the following integrodifferential model of mutualism: $d N_{1}(t) / d t=$ $r_{1} N_{1}(t)\left[\left(\left(K_{1}+\alpha_{1} \int_{0}^{\infty} J_{2}(s) N_{2}(t-s) d s\right) /\left(1+\int_{0}^{\infty} J_{2}(s) N_{2}(t-s) d s\right)\right)-N_{1}(t)\right], d N_{2}(t) / d t=r_{2} N_{2}(t)\left[\left(\left(K_{2}+\alpha_{2} \int_{0}^{\infty} J_{1}(s) N_{1}(t-s) d s\right) /(1+\right.\right.$ $\left.\left.\left.\int_{0}^{\infty} J_{1}(s) N_{1}(t-s) d s\right)\right)-N_{2}(t)\right]$, where $r_{i}, K_{i}$, and $\alpha_{i}, i=1,2$, are all positive constants. Consider $\alpha_{i}>K_{i}, i=1,2$. Consider $J_{i} \in C([0,+\infty),[0,+\infty))$ and $\int_{0}^{\infty} J_{i}(s) d s=1, i=1,2$. Our result shows that conditions which ensure the permanence of the system are enough to ensure the global stability of the system. The result not only improves but also complements some existing ones.

\section{Introduction}

Mutualism, one of the most important relationships in the theory of ecology, however, was pointed out by Murray as follows [1]: "this area has not been as widely sutdied as the others even though its importance is comparable to that of predator-prey and competition interactions."

Traditional two species Lotka-Volterra take the form

$$
\begin{aligned}
& \frac{d N_{1}}{d t}=r_{1} N_{1}\left(1-\frac{N_{1}}{K_{1}}+b_{12} \frac{N_{2}}{K_{1}}\right), \\
& \frac{d N_{2}}{d t}=r_{2} N_{2}\left(1-\frac{N_{2}}{K_{2}}+b_{21} \frac{N_{1}}{K_{2}}\right) .
\end{aligned}
$$

Murray [1] gave detail analysis of the phase trajectories for the above system. He also pointed out that the system has certain drawback; one is the sensitivity between unbounded growth and a finite positive steady state. Despite the drawback of the system, since it is the most simple model on the mutualism, scholars incorporated delays to the above system and proposed the following system:

$$
\begin{aligned}
& \frac{d x_{1}}{d t}=x_{1}(t)\left[r_{1}-a_{11} x_{1}\left(t-\tau_{11}\right)+a_{12} x_{2}\left(t-\tau_{12}\right)\right], \\
& \frac{d x_{2}}{d t}=x_{2}(t)\left[r_{2}+a_{21} x_{1}\left(t-\tau_{21}\right)-a_{22} x_{2}\left(t-\tau_{22}\right)\right] .
\end{aligned}
$$

Chen et al. [2] had given two examples to show that under the assumption $a_{11} a_{22}>a_{12} a_{21}$, a condition which could ensure the global stability of the system without delay, the system still admits unbounded solution. He and Gopalsamy [3] and Mukherjee [4] tried to investigate the persistent and stability property of the nonautonomous case of above system; however, in their main results, in addition to condition $a_{11} a_{22}>$ $a_{12} a_{21}$, they further assumed that the density of one of the species should be bounded from above, such an assumption is by no means easy to verify. To overcome this difficulty, Lu et al. [5, 6] and Nakata and Muroya [7] tried to give restriction on the coefficients of the system or restriction on the delay of the system, and some interesting results about the permanence of Lotka-Volterra type mutualism system with delay were obtained. Liu et al. [8] and $\mathrm{Lu}$ [9] also investigated the positive periodic solution of the Lotka-Volterra type mutualism model.

On the other hand, stimulated by the functional response of the predator-prey system, Wright [10] proposed the following two species mutualism model:

$$
\begin{aligned}
& \frac{d N}{d t}=r_{1} N\left[K_{1}-c_{1} N+\frac{b_{1} a_{1} M}{1+a_{1} T_{h 1} M}\right], \\
& \frac{d M}{d t}=r_{2} M\left[K_{2}-c_{2} N+\frac{b_{2} a_{2} N}{1+a_{2} T_{h 2} N}\right] .
\end{aligned}
$$


Obviously, the model could be revised as follows:

$$
\begin{aligned}
& \frac{d N(t)}{d t}=r_{1} N\left[\frac{K_{1}+\alpha_{1} M}{1+a_{1} T_{h 1} M}-c_{1} N\right], \\
& \frac{d M(t)}{d t}=r_{2} M\left[\frac{K_{2}+\alpha_{2} N}{1+a_{2} T_{h 2} N}-c_{2} M\right],
\end{aligned}
$$

where $\alpha_{1}=K_{1}+a_{1} T_{h 1}, \alpha_{2}=K_{2}+a_{2} T_{h 2}$, and one could easily see that $\alpha_{i}>K_{i}, i=1,2$.

It is well known that in a more realistic model the delay effect should be an average over past populations. This results in an equation with a distributed delay or an infinite delay. Based on the model (4), Gopalsamy [11] further proposed the following two species mutualism model:

$$
\begin{aligned}
& \frac{d N_{1}(t)}{d t}=r_{1} N_{1}\left[\frac{K_{1}+\alpha_{1} \int_{0}^{\infty} K_{2}(s) N_{2}(t-s) d s}{1+\int_{0}^{\infty} K_{2}(s) N_{2}(t-s) d s}-N_{1}(t)\right], \\
& \frac{d N_{2}(t)}{d t}=r_{2} N_{2}\left[\frac{K_{2}+\alpha_{2} \int_{0}^{\infty} K_{1}(s) N_{1}(t-s) d s}{1+\int_{0}^{\infty} K_{1}(s) N_{1}(t-s) d s}-N_{2}(t)\right] .
\end{aligned}
$$

However, the author did not investigate the dynamic behaviors of the system.

Recently, Li and $\mathrm{Xu}$ [12] proposed and studied the following nonautonomous case of the system (5):

$$
\begin{aligned}
& \frac{d N_{1}(t)}{d t} \\
& =r_{1}(t) N_{1}(t) \\
& \times\left[\frac{K_{1}(t)+\alpha_{1}(t) \int_{0}^{\infty} J_{2}(s) N_{2}(t-s) d s}{1+\int_{0}^{\infty} J_{2}(s) N_{2}(t-s) d s}-N_{1}(t)\right], \\
& \frac{d N_{2}(t)}{d t} \\
& =r_{2}(t) N_{2}(t) \\
& \times\left[\frac{K_{2}(t)+\alpha_{2}(t) \int_{0}^{\infty} J_{1}(s) N_{1}(t-s) d s}{1+\int_{0}^{\infty} J_{1}(s) N_{1}(t-s) d s}-N_{2}(t)\right],
\end{aligned}
$$

where $r_{i}, K_{i}, \alpha_{i}$, and $\sigma_{i}, i=1,2$, are continuous functions bounded above and below by positive constants. Consider $\alpha_{i}>K_{i}, i=1,2$. Consider $J_{i} \in C([0,+\infty),[0,+\infty))$ and $\int_{0}^{\infty} J_{i}(s) d s=1, i=1,2$. Under the assumption that $r_{i}$, $K_{i}$, and $\alpha_{i}, i=1,2$, are continuous periodic functions with common period $\omega . \alpha_{i}>K_{i}, i=1,2, J_{i} \in C([0,+\infty),[0,+\infty))$ and $\int_{0}^{\infty} J_{i}(s) d s=1, i=1,2$. By applying the coincidence degree theory, they showed that system (6) admits at least one positive $\omega$-periodic solution. Chen and You [13] argued that the general nonautonomous case is more suitable. Concerned with the persistent property of the system (6), by applying an integral inequality (see Lemma 3 in the next section), they obtained the following result.
Theorem A. The system (6) is always permanent. That is, there exist constants $m_{i}, M_{i}, i=1,2$, which are independent of the solution of the system (6), such that

$$
m_{i} \leq \liminf _{t \rightarrow+\infty} N_{i}(t) \leq \limsup _{t \rightarrow+\infty} N_{i}(t) \leq M_{i}, \quad i=1,2 .
$$

Such a result is a roughly one, since it only tells us that the solution is finally bounded above and below by positive constants and there is no fine description of the stable or unstable property of the solution, for example, whether the delay of the system could induce the Hopf bifurcation to period solution or not? Does the system admit some kind of chaotic behaviors? Is it difficult to obtain sufficient conditions which ensure the global attractivity of the positive solution? Indeed, to the best of the authors' knowledge, to this day, still no scholars investigate the stability property of the system (6), which is one of the most important topics in the study of population dynamics. Noting that system (6) is nonautonomous one and for such kind of system, generally speaking, by constructing some suitable Lyapunov functional, one could always obtain some sufficient conditions which ensure the stability of the system; however, the condition is not easy to verify [14]. This motivated us to investigate the stability property of the system (5).

From the point of view of biology, in the sequel, we will consider (5) together with the initial conditions

$$
N_{i}(s)=\phi_{i}(s), \quad s \in(-\infty, 0], i=1,2,
$$

where $\phi_{i} \in B C^{+}$and

$$
\begin{array}{r}
B C^{+}=\{\phi \in C((-\infty, 0],[0,+\infty)): \\
\phi(0)>0, \phi \text { be bounded }\} \\
i=1,2 .
\end{array}
$$

From [15], system (5) has a unique positive solution $\left(N_{1}(t), N_{2}(t)\right)$ satisfying the initial condition (8).

The aim of this paper is, by further developing the analysis technique of Chen and You [13] and de Oca and Vivas [16] and using the differential inequality theory, to obtain a set of sufficient conditions to ensure the global attractivity of the system (5). More precisely, we will prove the following result.

Theorem 1. System (5) admits a unique positive equilibrium $\left(N_{1}^{*}, N_{2}^{*}\right)$, which is globally attractive; that is, for any positive solution $\left(N_{1}(t), N_{2}(t)\right)$ of system (5) with the initial condition (8), one has

$$
\lim _{t \rightarrow+\infty} N_{i}(t)=N_{i}^{*}, \quad i=1,2 .
$$

We will prove this theorem in the next section and then give a brief discussion in Section 3. For more works on the mutualism or cooperation system, one could refer to [12-15, 17-21] and the references cited therein.

\section{Proof of the Main Result}

Now let us state several lemmas which will be useful in the proving of main result. 
Lemma 2. System (5) admits a unique positive equilibrium $\left(N_{1}^{*}, N_{2}^{*}\right)$.

Proof. The positive equilibrium of the system (5) satisfies the following equation:

$$
\begin{aligned}
& \frac{K_{1}+\alpha_{1} N_{2}}{1+N_{2}}-N_{1}=0, \\
& \frac{K_{2}+\alpha_{2} N_{1}}{1+N_{1}}-N_{2}=0 .
\end{aligned}
$$

System (11) admits a unique positive solution $\left(N_{1}^{*}, N_{2}^{*}\right)$, where

$$
\begin{gathered}
N_{1}^{*}=\frac{-A_{2}+\sqrt{A_{2}^{2}-4 A_{1} A_{3}}}{2 A_{1}}, \\
N_{2}^{*}=\frac{-B_{2}+\sqrt{B_{2}^{2}-4 B_{1} B_{3}}}{2 B_{1}}, \\
A_{1}=1+\alpha_{2}, \\
A_{2}=K_{2}-K_{1}-\alpha_{2} \alpha_{1}+1, \\
A_{3}=-\alpha_{1} K_{2}-K_{1}, \\
B_{1}=1+\alpha_{1}, \\
B_{2}=K_{1}-K_{2}-\alpha_{1} \alpha_{2}+1, \\
B_{3}=-K_{2}-\alpha_{2} K_{1} .
\end{gathered}
$$

This ends the proof of Lemma 2.

Following Lemma 3 is Lemma 3 of de Oca and Vivas [16].

Lemma 3. Let $x: R \rightarrow R$ be a bounded nonnegative continuous function, and let $k:[0,+\infty) \rightarrow(0,+\infty)$ be a continuous kernel such that $\int_{0}^{\infty} k(s) d s=1$. Then

$$
\begin{aligned}
\liminf _{t \rightarrow+\infty} x(t) & \leq \liminf _{t \rightarrow+\infty} \int_{-\infty}^{t} k(t-s) x(s) d s \\
& \leq \limsup _{t \rightarrow+\infty} \int_{-\infty}^{t} k(t-s) x(s) d s \\
& \leq \limsup _{t \rightarrow+\infty} x(t) .
\end{aligned}
$$

As a direct corollary of Lemma 2.2 of Chen [22], we have the following lemma.

Lemma 4. If $a>0, b>0$, and $\dot{x} \geq x(b-a x)$, when $t \geq 0$ and $x(0)>0$, one has

$$
\liminf _{t \rightarrow+\infty} x(t) \geq \frac{b}{a} .
$$

If $a>0, b>0$ and $\dot{x} \leq x(b-a x)$, when $t \geq 0$ and $x(0)>0$, one has

$$
\limsup _{t \rightarrow+\infty} x(t) \leq \frac{b}{a}
$$

Now we are in the position of proving the main result of this paper.

Proof of Theorem 1. Let $\left(N_{1}(t), N_{2}(t)\right)$ be any positive solution of the system (5) with initial condition (8). Similarly to the analysis of (11)-(17) in [13], from the first equation of the system (5) it follows that

$$
\frac{d N_{1}(t)}{d t} \leq N_{1}(t)\left[r_{1}\left(K_{1}+\alpha_{1}\right)-r_{1} N_{1}(t)\right] .
$$

Thus, as a direct corollary of Lemma 4, according to (16), one has

$$
\limsup _{t \rightarrow+\infty} N_{1}(t) \leq K_{1}+\alpha_{1}
$$

and so, from Lemma 3 we have

$$
\limsup _{t \rightarrow+\infty} \int_{0}^{\infty} J_{1}(s) N_{1}(t-s) d s \leq K_{1}+\alpha_{1} .
$$

Hence, for enough small $\varepsilon>0$, it follows from (17) and (18) that there exists a $T_{1}^{\prime}>0$ such that

$$
\begin{gathered}
N_{1}(t)<K_{1}+\alpha_{1}+\varepsilon \stackrel{\text { def }}{=} M_{1}^{(1)} \\
\int_{0}^{\infty} J_{1}(s) N_{1}(t-s) d s=\int_{-\infty}^{t} J_{1}(t-s) N_{1}(s) d s \\
\leq K_{1}+\alpha_{1}+\varepsilon \stackrel{\text { def }}{=} M_{1}^{(1)} \text { for } t>T_{1}^{\prime} .
\end{gathered}
$$

Similarly, for above $\varepsilon>0$, it follows from the second equation of the system (5) that there exists a $T_{1}>T_{1}^{\prime}$ such that

$$
\begin{gathered}
N_{2}(t)<K_{2}+\alpha_{2}+\varepsilon \stackrel{\text { def }}{=} M_{2}^{(1)} \\
\int_{0}^{\infty} J_{2}(s) N_{2}(t-s) d s<K_{2}+\alpha_{2}+\varepsilon \stackrel{\text { def }}{=} M_{2}^{(1)}
\end{gathered}
$$

for $t>T_{1}$.

Noting that the function $g_{1}(x)=\left(\left(K_{1}+\alpha_{1} x\right) /(1+x)\right)\left(\alpha_{1}>\right.$ $\left.K_{1}, x \geq 0\right)$ is a strictly increasing function, hence, (20) together with the first equation of the system (5) implies

$$
\begin{array}{r}
\frac{d N_{1}(t)}{d t}<N_{1}(t)\left[\frac{r_{1}\left(K_{1}+\alpha_{1} M_{2}^{(1)}\right)}{1+M_{2}^{(1)}}-r_{1} N_{1}(t)\right] \\
\text { for } t>T_{1} .
\end{array}
$$

Therefore, by Lemma 4, we have

$$
\limsup _{t \rightarrow+\infty} N_{1}(t) \leq \frac{K_{1}+\alpha_{1} M_{2}^{(1)}}{1+M_{2}^{(1)}} .
$$

Thus, from Lemma 3 we have

$$
\limsup _{t \rightarrow+\infty} \int_{0}^{\infty} J_{1}(s) N_{1}(t-s) d s \leq \frac{K_{1}+\alpha_{1} M_{2}^{(1)}}{1+M_{2}^{(1)}} .
$$


That is, for $\varepsilon>0$ defined by (19), there exists a $T_{2}^{\prime}>T_{1}$ such that

$$
\begin{gathered}
N_{1}(t)<\frac{K_{1}+\alpha_{1} M_{2}^{(1)}}{1+M_{2}^{(1)}}+\frac{\varepsilon}{2} \stackrel{\text { def }}{=} M_{1}^{(2)}>0, \\
\int_{0}^{\infty} J_{1}(s) N_{1}(t-s) d s<\frac{K_{1}+\alpha_{1} M_{2}^{(1)}}{1+M_{2}^{(1)}}+\frac{\varepsilon}{2} \stackrel{\text { def }}{=} M_{1}^{(2)}>0
\end{gathered}
$$$$
\text { for } t>T_{2}^{\prime} \text {. }
$$

Similarly to the analysis of (22)-(24), from (19) and the second equation of the system (5), there exists a $T_{2}>T_{2}^{\prime}$ such that

$$
\begin{gathered}
N_{2}(t)<\frac{K_{2}+\alpha_{2} M_{1}^{(1)}}{1+M_{1}^{(1)}}+\frac{\varepsilon}{2} \stackrel{\text { def }}{=} M_{2}^{(2)}>0, \\
\int_{0}^{\infty} J_{2}(s) N_{2}(t-s) d s<\frac{K_{2}+\alpha_{2} M_{1}^{(1)}}{1+M_{1}^{(1)}}+\frac{\varepsilon}{2} \stackrel{\text { def }}{=} M_{2}^{(2)}>0 \\
\quad \text { for } t>T_{2} .
\end{gathered}
$$

Since the function $g_{1}(x)=\left(\left(K_{1}+\alpha_{1} x\right) /(1+x)\right)\left(\alpha_{1}>K_{1}\right.$, $x \geq 0)$ is a strictly increasing function, one could easily see that $g(x) \geq g(0)=K_{1}$, and so, from the first equation of the system (5), it follows that

$$
\frac{d N_{1}(t)}{d t} \geq N_{1}(t)\left[r_{1} K_{1}-r_{1} N_{1}(t)\right]
$$

Thus, as a direct corollary of Lemma 4, according to (25), one has

$$
\liminf _{t \rightarrow+\infty} N_{1}(t) \geq K_{1}
$$

and so, from Lemma 3, we have

$$
\liminf _{t \rightarrow+\infty} \int_{0}^{\infty} J_{1}(s) N_{1}(t-s) d s \geq K_{1}
$$

Hence, for enough small $\varepsilon>0\left(\varepsilon<(1 / 2) \min \left\{K_{1}, K_{2}\right\}\right)$, it follows from (27) and (28) that there exists a $T_{3}^{\prime}>0$ such that

$$
\begin{gathered}
N_{1}(t)>K_{1}-\varepsilon \stackrel{\text { def }}{=} m_{1}^{(1)}, \\
\int_{0}^{\infty} J_{1}(s) N_{1}(t-s) d s \geq K_{1}-\varepsilon \stackrel{\text { def }}{=} m_{1}^{(1)}
\end{gathered}
$$

$$
\text { for } t>T_{3}^{\prime} \text {. }
$$

Similarly, for above $\varepsilon>0$, it follows from the second equation of the system (5) that there exists a $T_{3}>T_{3}^{\prime}$ such that

$$
\begin{gathered}
N_{2}(t)>K_{2}-\varepsilon \stackrel{\text { def }}{=} m_{2}^{(1)}, \\
\int_{0}^{\infty} J_{2}(s) N_{2}(t-s) d s>K_{2}-\varepsilon \stackrel{\text { def }}{=} m_{2}^{(1)}
\end{gathered}
$$

for $t>T_{3}$.
Noting that the function $g_{1}(x)=\left(\left(K_{1}+\alpha_{1} x\right) /(1+x)\right)\left(\alpha_{1}>\right.$ $\left.K_{1}\right)$ is a strictly increasing function, hence, (30) together with the first equation of the system (5) implies

$$
\begin{array}{r}
\frac{d N_{1}(t)}{d t}>N_{1}(t)\left[\frac{r_{1}\left(K_{1}+\alpha_{1} m_{2}^{(1)}\right)}{1+m_{2}^{(1)}}-r_{1} N_{1}(t)\right] \\
\text { for } t>T_{3} .
\end{array}
$$

Therefore, by Lemma 4, we have

$$
\liminf _{t \rightarrow+\infty} N_{1}(t) \geq \frac{K_{1}+\alpha_{1} m_{2}^{(1)}}{1+m_{2}^{(1)}} .
$$

Thus, from Lemma 3 we have

$$
\liminf _{t \rightarrow+\infty} \int_{0}^{\infty} J_{1}(s) N_{1}(t-s) d s \geq \frac{K_{1}+\alpha_{1} m_{2}^{(1)}}{1+m_{2}^{(1)}} .
$$

That is, there exists a $T_{4}^{\prime}>T_{3}$ such that

$$
\begin{gathered}
N_{1}(t)>\frac{K_{1}+\alpha_{1} m_{2}^{(1)}}{1+m_{2}^{(1)}}-\frac{\varepsilon}{2} \stackrel{\text { def }}{=} m_{1}^{(2)}>0, \\
\int_{0}^{\infty} J_{1}(s) N_{1}(t-s) d s>\frac{K_{1}+\alpha_{1} m_{2}^{(1)}}{1+m_{2}^{(1)}}-\frac{\varepsilon}{2} \stackrel{\text { def }}{=} m_{1}^{(2)}>0 \\
\\
\quad \text { for } t>T_{4}^{\prime} .
\end{gathered}
$$

Similarly to the analysis of (32)-(34), from (28) and the second equation of the system (5), there exists a $T_{4}>T_{4}^{\prime}$ such that

$$
\begin{gathered}
N_{2}(t)>\frac{K_{2}+\alpha_{2} m_{1}^{(1)}}{1+m_{1}^{(1)}}-\frac{\varepsilon}{2} \stackrel{\text { def }}{=} m_{2}^{(2)}>0, \\
\int_{0}^{\infty} J_{2}(s) N_{2}(t-s) d s>\frac{K_{2}+\alpha_{2} m_{1}^{(1)}}{1+m_{1}^{(1)}}-\frac{\varepsilon}{2} \stackrel{\text { def }}{=} m_{2}^{(2)}>0 \\
\text { for } t>T_{4} .
\end{gathered}
$$

One could easily see that

$$
\begin{gathered}
M_{1}^{(2)}=\frac{K_{1}+\alpha_{1} M_{2}^{(1)}}{1+M_{2}^{(1)}}+\frac{\varepsilon}{2}<K_{1}+\alpha_{1}+\varepsilon=M_{1}^{(1)} \\
M_{2}^{(2)}=\frac{K_{2}+\alpha_{2} M_{1}^{(1)}}{1+M_{1}^{(1)}}+\frac{\varepsilon}{2}<K_{2}+\alpha_{2}+\varepsilon=M_{2}^{(1)} \\
m_{1}^{(2)}=\frac{K_{1}+\alpha_{1} m_{2}^{(1)}}{1+m_{2}^{(1)}}-\frac{\varepsilon}{2}>K_{1}-\varepsilon=m_{1}^{(1)} \\
m_{2}^{(2)}=\frac{K_{2}+\alpha_{2} m_{1}^{(1)}}{1+m_{1}^{(1)}}-\frac{\varepsilon}{2}>K_{2}-\varepsilon=m_{2}^{(1)} .
\end{gathered}
$$


Repeating the above procedure, we get four sequences $M_{i}^{(n)}$, $m_{i}^{(n)}, i=1,2, n=1,2, \ldots$, such that for $n \geq 2$

$$
\begin{aligned}
& M_{1}^{(n)}=\frac{K_{1}+\alpha_{1} M_{2}^{(n-1)}}{1+M_{2}^{(n-1)}}+\frac{\varepsilon}{n} ; \\
& M_{2}^{(n)}=\frac{K_{2}+\alpha_{2} M_{1}^{(n-1)}}{1+M_{1}^{(n-1)}}+\frac{\varepsilon}{n} ; \\
& m_{1}^{(n)}=\frac{K_{1}+\alpha_{1} m_{2}^{(n-1)}}{1+m_{2}^{(n-1)}}-\frac{\varepsilon}{n} ; \\
& m_{2}^{(n)}=\frac{K_{2}+\alpha_{2} m_{1}^{(n-1)}}{1+m_{1}^{(n-1)}}-\frac{\varepsilon}{n} .
\end{aligned}
$$

Obviously,

$$
m_{i}^{(n)}<N_{i}(t)<M_{i}^{(n)}, \quad \text { for } t \geq T_{2 n}, \quad i=1,2 .
$$

We claim that sequences $M_{i}^{(n)}, i=1,2$ are nonincreasing and sequences $m_{i}^{(n)}, i=1,2$ are nondecreasing. To prove this claim, we will carry out by induction. Firstly, from (36) we have

$$
M_{i}^{(2)}<M_{i}^{(1)}, \quad m_{i}^{(2)}>m_{i}^{(1)}, \quad i=1,2 .
$$

Let us assume now that our claim is true for $n$; that is,

$$
M_{i}^{(n)}<M_{i}^{(n-1)}, \quad m_{i}^{(n)}>m_{i}^{(n-1)}, \quad i=1,2 .
$$

Again from the strict increasing of the function $g_{i}(x)=\left(\left(K_{i}+\right.\right.$ $\left.\left.\alpha_{i} x\right) /(1+x)\right)\left(\alpha_{i}>K_{i}, i=1,2\right)$, we immediately obtain

$$
\begin{aligned}
M_{1}^{(n+1)} & =\frac{K_{1}+\alpha_{1} M_{2}^{(n)}}{1+M_{2}^{(n)}}+\frac{\varepsilon}{n+1} \\
& <\frac{K_{1}+\alpha_{1} M_{2}^{(n-1)}}{1+M_{2}^{(n-1)}}+\frac{\varepsilon}{n}=M_{1}^{(n)} ; \\
M_{2}^{(n+1)} & =\frac{K_{2}+\alpha_{2} M_{1}^{(n)}}{1+M_{1}^{(n)}}+\frac{\varepsilon}{n+1} \\
& <\frac{K_{2}+\alpha_{2} M_{1}^{(n-1)}}{1+M_{1}^{(n-1)}}+\frac{\varepsilon}{n}=M_{2}^{(n)} ; \\
m_{1}^{(n+1)} & =\frac{K_{1}+\alpha_{1} m_{2}^{(n)}}{1+m_{2}^{(n)}-\frac{\varepsilon}{n+1}} \\
> & \frac{K_{1}+\alpha_{1} m_{2}^{(n-1)}}{1+m_{2}^{(n-1)}}-\frac{\varepsilon}{n}=m_{1}^{(n)} ; \\
m_{2}^{(n+1)} & =\frac{K_{2}+\alpha_{2} m_{1}^{(n)}}{1+m_{1}^{(n)}}-\frac{\varepsilon}{n+1} \\
& \frac{K_{2}+\alpha_{2} m_{1}^{(n-1)}}{1+m_{1}^{(n-1)}}-\frac{\varepsilon}{n}=m_{2}^{(n)}
\end{aligned}
$$

Therefore,

$$
\lim _{t \rightarrow+\infty} M_{i}^{(n)}=\bar{N}_{i}, \quad \lim _{t \rightarrow+\infty} m_{i}^{(n)}=\underline{N}_{i}, \quad i=1,2 .
$$

Letting $n \rightarrow+\infty$ in (37), we obtain

$$
\begin{array}{ll}
\bar{N}_{1}=\frac{K_{1}+\alpha_{1} \bar{N}_{2}}{1+\bar{N}_{2}}, & \bar{N}_{2}=\frac{K_{2}+\alpha_{2} \bar{N}_{1}}{1+\bar{N}_{1}}, \\
\underline{N}_{1}=\frac{K_{1}+\alpha_{1} \underline{N}_{2}}{1+\underline{N}_{2}}, & \underline{N}_{2}=\frac{K_{2}+\alpha_{2} \underline{N}_{1}}{1+\underline{N}_{1}}
\end{array}
$$

and (43) shows that $\left(\bar{N}_{1}, \bar{N}_{2}\right)$ and $\left(\underline{N}_{1}, \underline{N}_{2}\right)$ are solutions of (11). By Lemma 2, (11) has a unique positive solution $E^{*}\left(N_{1}^{*}, N_{2}^{*}\right)$. Hence, we conclude that

$$
\bar{N}_{i}=\underline{N}_{i}=N_{i}^{*}, \quad i=1,2 ;
$$

that is,

$$
\lim _{t \rightarrow+\infty} N_{i}(t)=N_{i}^{*} \quad i=1,2 .
$$

Thus, the unique interior equilibrium $E^{*}\left(N_{1}^{*}, N_{2}^{*}\right)$ is globally attractive. This completes the proof of Theorem 1.

\section{Discussion}

As was pointed out in the introduction section, for LotkaVolterra type mutualism system, conditions which ensure the globally stability of the system are not so easily verified $[3,4]$. In this paper, we study the stability property of the integrodifferential model of mutualism (5). By applying the iterative technique, we obtain a set of sufficient conditions which guarantee the global attractivity of the coexistence equilibrium. Our result (Theorem 1) shows that the conditions which ensure the permanence of the system are enough to ensure the global attractivity of the system. The new finding of this paper is that we found the iterative bound of the solution, such a finding is not detected by Chen and You [13]. Based on this finding, it is possible to obtain the subtle result about the stability of the system. We mention here that we did not consider the delay in the intraspecific competition; whether such kind of delay could induce bifurcation or not is still unknown; we leave this for future study.

\section{Conflict of Interests}

The authors declare that there is no conflict of interests regarding the publication of this paper.

\section{Acknowledgments}

The authors are grateful to anonymous referees for their excellent suggestions, which greatly improved the presentation of the paper. Also, this work was supported by the Natural Science Foundation of Fujian Province (2013J01011 and 2013J01010) and the Foundation of Fujian Education Bureau (JA13361). 


\section{References}

[1] J. D. Murray, Mathematical Biology, Springer, Berlin, Germany, 1998.

[2] L. S. Chen, Z. Y. Lu, and W. D. Wang, "The effect of delays on the permanence for Lotka-Volterra systems," Applied Mathematics Letters, vol. 8, no. 4, pp. 71-73, 1995.

[3] X.-z. He and K. Gopalsamy, "Persistence, attractivity, and delay in facultative mutualism," Journal of Mathematical Analysis and Applications, vol. 215, no. 1, pp. 154-173, 1997.

[4] D. Mukherjee, "Permanence and global attractivity for facultative mutualism system with delay," Mathematical Methods in the Applied Sciences, vol. 26, no. 1, pp. 1-9, 2003.

[5] G. Lu, Z. Lu, and X. Lian, "Delay effect on the permanence for Lotka-Volterra cooperative systems," Nonlinear Analysis: Real World Applications, vol. 11, no. 4, pp. 2810-2816, 2010.

[6] G. Lu and Z. Lu, "Permanence for two-species Lotka-Volterra cooperative systems with delays," Mathematical Biosciences and Engineering, vol. 5, no. 3, pp. 477-484, 2008.

[7] Y. Nakata and Y. Muroya, "Permanence for nonautonomous Lotka-Volterra cooperative systems with delays," Nonlinear Analysis: Real World Applications, vol. 11, no. 1, pp. 528-534, 2010.

[8] Z. Liu, R. Tan, Y. Chen, and L. Chen, "On the stable periodic solutions of a delayed two-species model of facultative mutualism," Applied Mathematics and Computation, vol. 196, no. 1, pp. 105-117, 2008.

[9] S. Lu, "On the existence of positive periodic solutions to a Lotka Volterra cooperative population model with multiple delays," Nonlinear Analysis: Theory, Methods \& Applications, vol. 68, no. 6, pp. 1746-1753, 2008.

[10] D. H. Wright, "A simple, stable model of mutualism incorporating handling time," The American Naturalist, vol. 134, no. 4, pp. 664-667, 1989.

[11] K. Gopalsamy, Stability and Oscillations in Delay Differential Equations of Population Dynamics, vol. 74 of Mathematics and Its Applications, Kluwer Academic, Boston, Mass, USA, 1992.

[12] Y. $\mathrm{Li}$ and G. Xu, "Positive periodic solutions for an integrodifferential model of mutualism," Applied Mathematics Letters, vol. 14, no. 5, pp. 525-530, 2001.

[13] F. Chen and M. You, "Permanence for an integrodifferential model of mutualism," Applied Mathematics and Computation, vol. 186, no. 1, pp. 30-34, 2007.

[14] F. Chen, X. Liao, and Z. Huang, "The dynamic behavior of $N$ species cooperation system with continuous time delays and feedback controls," Applied Mathematics and Computation, vol. 181, no. 2, pp. 803-815, 2006.

[15] J. Hale, Theory of Functional Differential Equations, Springer, Heidelberg, Germany, 2nd edition, 1977.

[16] F. M. de Oca and M. Vivas, "Extinction in two dimensional Lotka-Volterra system with infinite delay," Nonlinear Analysis: Real World Applications, vol. 7, no. 5, pp. 1042-1047, 2006.

[17] F. Chen, J. Yang, L. Chen, and X. Xie, "On a mutualism model with feedback controls," Applied Mathematics and Computation, vol. 214, no. 2, pp. 581-587, 2009.

[18] L. Chen, L. Chen, and Z. Li, "Permanence of a delayed discrete mutualism model with feedback controls," Mathematical and Computer Modelling, vol. 50, no. 7-8, pp. 1083-1089, 2009.

[19] L. Chen and X. Xie, "Permanence of an $N$-species cooperation system with continuous time delays and feedback controls," Nonlinear Analysis: Real World Applications, vol. 12, no. 1, pp. 34-38, 2011.
[20] Y. Li and T. Zhang, "Permanence of a discrete $N$-species cooperation system with time-varying delays and feedback controls," Mathematical and Computer Modelling, vol. 53, no. 56, pp. 1320-1330, 2011.

[21] F. Y. Wei and C. Y. Li, "Permanence and globally asymptotic stability of cooperative system incorporating harvesting," Advances in Pure Mathematics, vol. 3, pp. 627-632, 2013.

[22] F. Chen, "On a nonlinear nonautonomous predator-prey model with diffusion and distributed delay," Journal of Computational and Applied Mathematics, vol. 180, no. 1, pp. 33-49, 2005. 


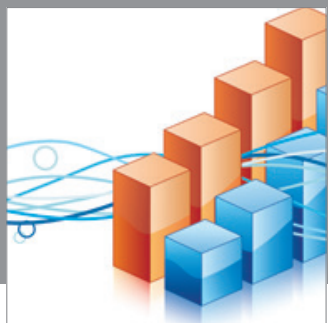

Advances in

Operations Research

mansans

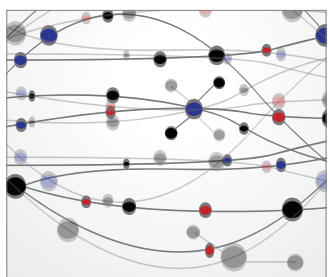

The Scientific World Journal
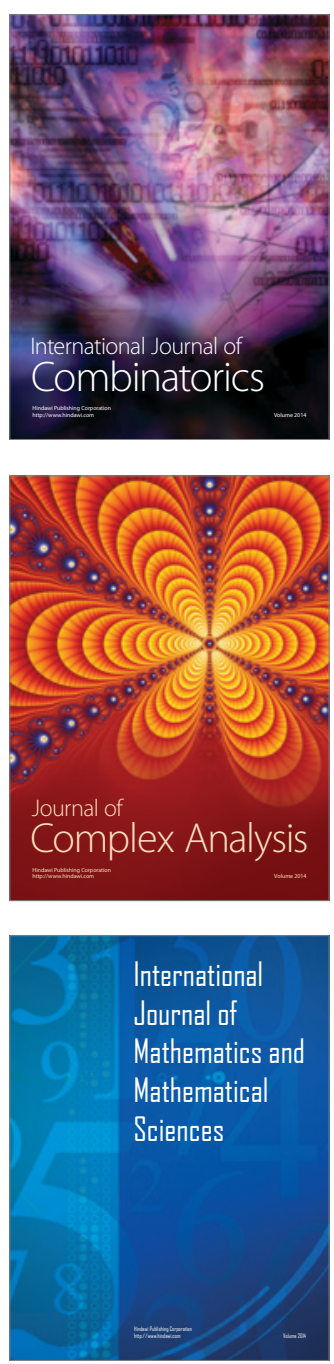
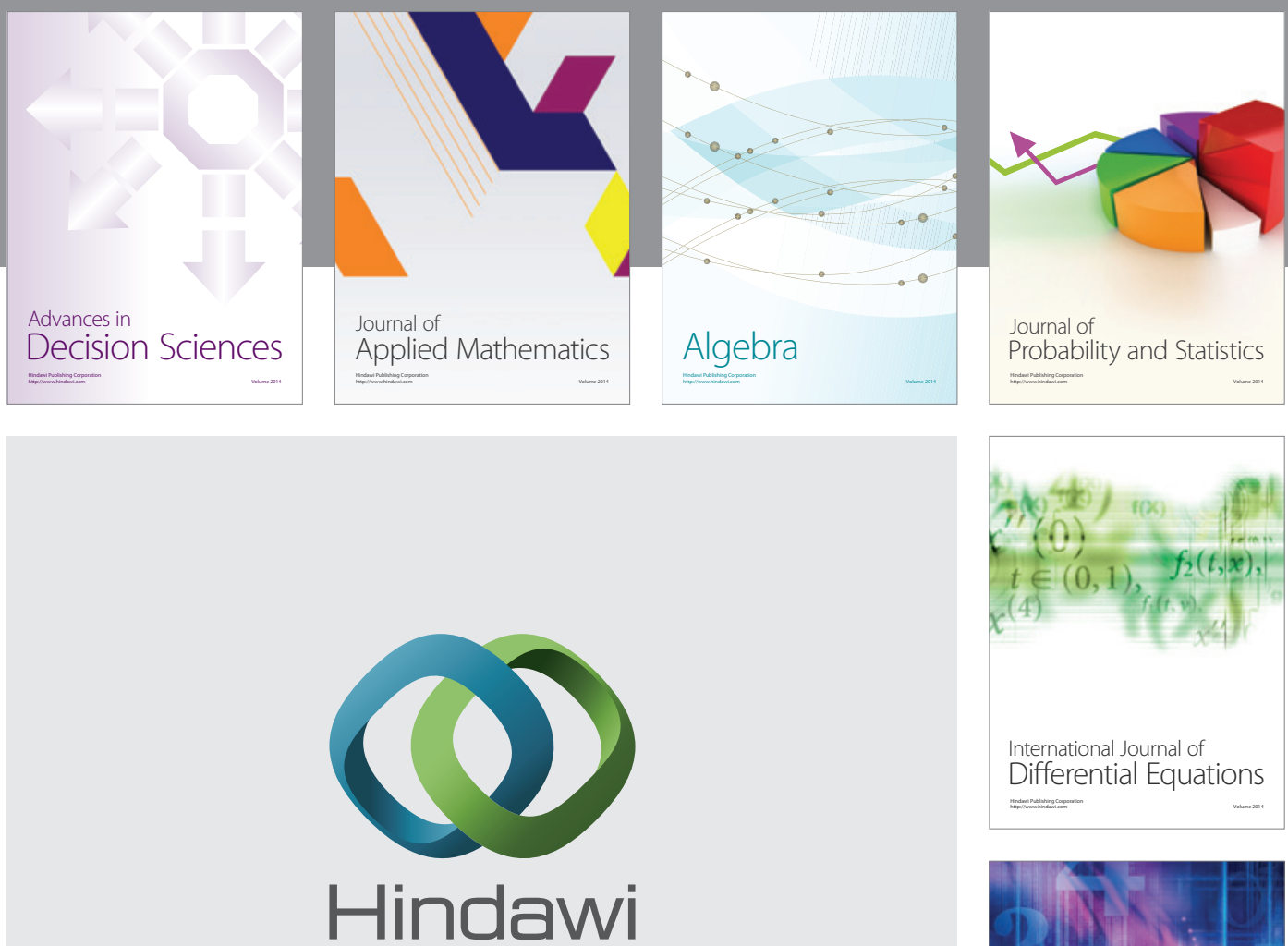

Submit your manuscripts at http://www.hindawi.com
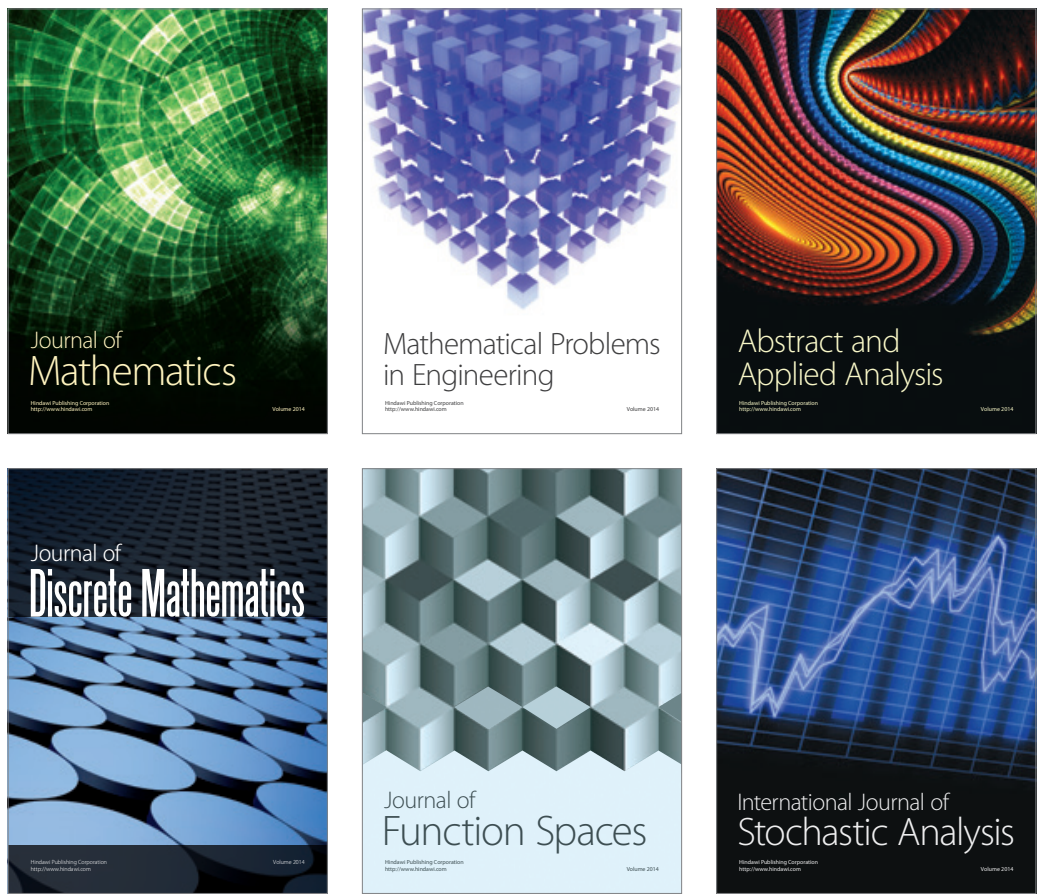

Journal of

Function Spaces

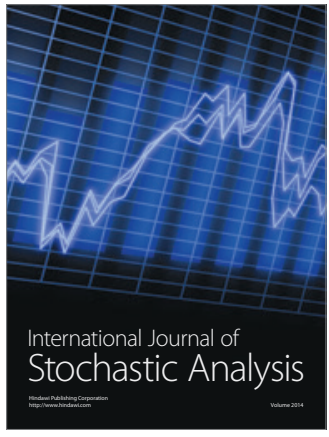

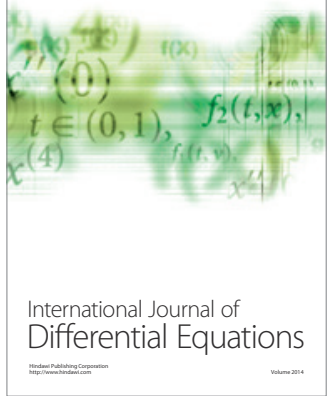
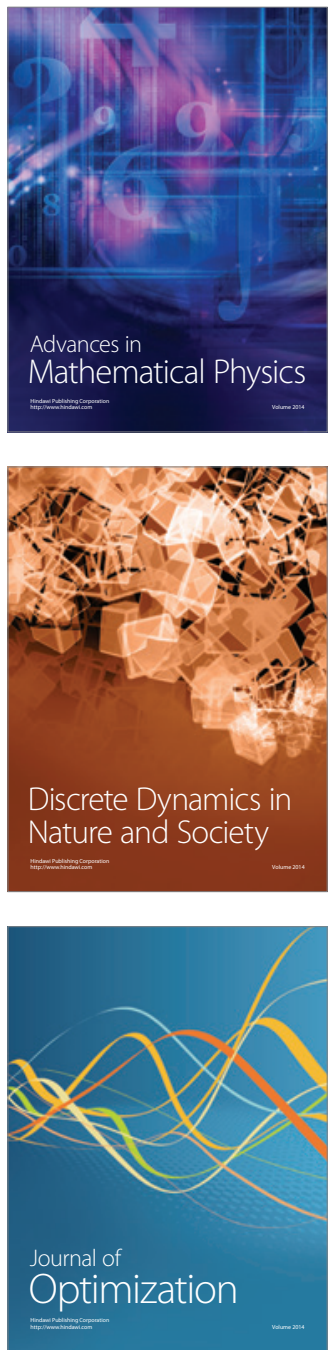